submitted Sept 12, 2006 to ApJ; accepted December 20, 2006

\title{
The Late-T Dwarf Companion to the Exoplanet Host Star HD 3651: A New Benchmark for Gravity and Metallicity Effects in Ultracool Spectra
}

\author{
Michael C. Liu, ${ }^{1,2,3}$ S. K. Leggett, ${ }^{4}$ Kuenley $\mathrm{Chiu}^{5}$
}

\begin{abstract}
We present near-IR $(1.0-2.5 \mu \mathrm{m})$ photometry and spectroscopy of HD 3651B, the low-luminosity, wide-separation (480 AU) companion to the K0V exoplanet host star HD 3651A. We measure a spectral type of T7.5 \pm 0.5 for HD 3651B, confirming both its substellar nature and the fact that wide-separation brown dwarfs and giant planets can co-exist around the same star. We estimate an age of 3-12 Gyr for the primary star HD $3651 \mathrm{~A}$ and find that it is $\approx 3 \times$ older than the K4V star Gl $570 \mathrm{~A}(\approx 1-5 \mathrm{Gyr})$, the host star of the T7.5 dwarf Gl 570D. We derive a bolometric luminosity of $\log \left(L_{\mathrm{bol}} / L_{\odot}\right)=$ $-5.58 \pm 0.05$ for HD $3651 \mathrm{~B}$ and infer an effective temperature of 780-840 $\mathrm{K}$ and a mass of 40-72 $M_{\mathrm{Jup}}$; the luminosity and temperature are among the lowest measured for any brown dwarf. Furthermore, HD 3651B belongs to the rare class of substellar objects that are companions to main-sequence stars and thus provides a new benchmark for studying very low-temperature objects. Given their similar temperatures $\left(\Delta T_{\text {eff }} \approx 30 \mathrm{~K}\right)$ and metallicities $(\Delta[\mathrm{Fe} / \mathrm{H}] \approx 0.1 \mathrm{dex})$ but different ages, a comparison of HD 3651B and Gl 570D allows us to examine gravity-sensitive diagnostics in ultracool spectra. We find that the expected signature of HD 3651B's higher surface gravity due to its older age, namely a suppressed $K$-band flux relative to Gl $570 \mathrm{D}$, is not seen. Instead, the $K$-band flux of HD 3651B is enhanced compared to Gl 570D, indicative of a younger age. Thus, the relative ages derived from interpretation of the $\mathrm{T}$ dwarf spectra and from stellar activity indicators appear to be in discord. One likely explanation is that the $K$-band fluxes are also very sensitive to metallicity differences. Metallicity variations may be as important as surface gravity variations in causing spectral differences among field late-T dwarfs.
\end{abstract}

Subject headings: stars: brown dwarfs — stars: atmospheres — infrared: stars

\footnotetext{
${ }^{1}$ Institute for Astronomy, University of Hawai‘i, 2680 Woodlawn Drive, Honolulu, HI 96822; mliu@ifa.hawaii.edu

${ }^{2}$ Alfred P. Sloan Research Fellow

${ }^{3}$ Visiting Astronomer at the Infrared Telescope Facility, which is operated by the University of Hawaii under Cooperative Agreement no. NCC 5-538 with the National Aeronautics and Space Administration, Science Mission Directorate, Planetary Astronomy Program.

${ }^{4}$ Gemini Observatory Northern Operations Center, 670 North A'ohoku Place, Hilo, HI 96720

${ }^{5}$ Astrophysics Group, School of Physics, University of Exeter, Stocker Road, Exeter, EX4 4QL, UK
} 


\section{Introduction}

Discovery and scrutiny of brown dwarfs have been fertile avenues for understanding selfluminous objects, extending traditional stellar astrophysics into new domains of mass and effective temperature. The coolest brown dwarfs, the $\mathrm{T}$ dwarfs, are characterized by very red optical colors from pressure-broadened alkali resonance lines and very blue near-IR colors from strong methane absorption and collision-induced $\mathrm{H}_{2}$ absorption (e.g. Oppenheimer et al. 1995; Geballe et al. 2002; Kirkpatrick 2005). T dwarfs are the lowest luminosity and coolest objects directly detected outside of our solar system, with bolometric luminosities $\left(L_{\mathrm{bol}}\right)$ of $\lesssim 10^{-4.5} L_{\odot}$ and effective temperatures $\left(T_{\text {eff }}\right)$ of $\approx 700-1300 \mathrm{~K}$ (e.g. Vrba et al. 2004; Golimowski et al. 2004; Burgasser et al. 2006a). As such, analyzing their spectra to infer temperature, gravity, metallicity, and mass is a key pathway to understanding the properties of gas-giant extrasolar planets.

The first T dwarf, Gl 229B, was discovered as a 45-AU companion around a nearby $\mathrm{M}$ dwarf (Nakajima et al. 1995). However, since then the vast majority have been found as free-floating field objects by the 2MASS and SDSS wide-field surveys (e.g. Burgasser et al. 1999; Leggett et al. 2000; Chiu et al. 2006), with about $100 \mathrm{~T}$ dwarfs identified to date. Such a populous sample has been a boon for probing substellar astrophysics. But this sample is also inevitably hindered by the unknown ages and metallicities of the field population. Binary brown dwarfs provide a partial solution to this challenge, as they constitute systems of common age and metallicity; however, the absolute values of these quantities are unknown for binaries. In this regard, brown dwarfs that are resolved companions to stars are highly prized, as their distances, ages and metallicities can be established from their primary stars, given the conservative assumption that the systems formed coevally and from material of the same composition. These brown dwarf companions serve as "benchmarks" for studying substellar atmospheres and evolution. Such objects are rare, as numerous published (and unpublished) imaging surveys have searched for brown dwarf companions to nearby main-sequence stars with very limited success (e.g. Lloyd et al. 2004; Oppenheimer et al. 2001; McCarthy \& Zuckerman 2004; Carson et al. 2006).

Mugrauer et al. (2006) have recently identified a low-luminosity companion to the nearby K0V star HD 3651 (GJ 27, 54 Psc, HR 166, HIP 3093, SAO 74175). The primary star is also notable as it possesses a sub-Saturn mass planet $\left(M \sin i=0.20 M_{\mathrm{Jup}}\right)$ with an orbital semi-major axis of $0.3 \mathrm{AU}$ (Fischer et al. 2003). HD 3651B has a projected separation of $43^{\prime \prime}$ (480 AU) and is confirmed to be physically associated by its common proper motion with HD 3651A. Given the distance of $11.11 \pm 0.09 \mathrm{pc}$ to the primary (Perryman et al. 1997), the very faint absolute $H$-band magnitude and the absence of an optical counterpart in photographic plates suggest that HD 3651B is a very cool brown dwarf.

In this paper, we present near-IR photometry and spectroscopy to characterize HD 3651B and compare it to Gl 570D, the coolest known companion around a main-sequence star. After

our paper was submitted, Luhman et al. (2006) reported identification and characterization of HD 3651B based on Spitzer/IRAC and ground-based near-IR measurements, and Burgasser (2006) 
reported near-IR spectroscopy and analysis.

\section{Observations}

\subsection{Photometry}

We obtained near-infrared (IR) imaging of HD 3651B on 2006 September 3 UT from the United Kingdom Infrared Telescope (UKIRT) located on Mauna Kea, Hawaii. We used the facility IR camera UFTI (Roche et al. 2003) and the $J(1.25 \mu \mathrm{m}), H(1.64 \mu \mathrm{m})$, and $K(2.20 \mu \mathrm{m})$ filters from the Mauna Kea Observatories (MKO) filter consortium (Simons \& Tokunaga 2002; Tokunaga et al. 2002). We read out a $512 \times 512$ pixel region of UFTI's detector centered on HD 3651B, leading to a $47^{\prime \prime}$ field of view. The primary star HD 3651A was placed off the array. UKIRT has a fast-steering secondary mirror that provides tip-tilt correction, producing an image quality of $0.55^{\prime \prime} \mathrm{FWHM}$ during our observations. HD 3651B appeared as a single object in the images. Sky conditions were photometric. We obtained a series of 9 dithered images in each filter, for a total on-source integration time of 9 minutes in $J, H$, and $K$ each. The images were reduced in a standard fashion using the facility data pipeline. The flux calibration was established from observations of

the UKIRT standard stars FS 154 and FS 102 (Leggett et al. 2006), observed immediately before and after HD 3651B and at similar airmass. Table 1 presents our final UKIRT/UFTI photometry.

\subsection{Spectroscopy}

We obtained spectroscopy of HD 3651B on 2006 September 4 and 5 UT using the UKIRT facility spectrograph CGS4 (Wright et al. 1993). CGS4 has a Santa Barbara Research Center (SBRC) $256 \times 256$ pixel InSb detector. The two-pixel $\left(1.2^{\prime \prime}\right)$ slit was used for all observations. HD $3651 \mathrm{~B}$ was observed at three grating settings to span the $J$-, $H$ - and $K$-bands, covering $1.03-$ $1.35,1.38-2.02$ and $1.88-2.52 \mu \mathrm{m}$ with spectral resolutions of $21 \AA$ at $J(R=\lambda / \Delta \lambda \approx 600)$ and $50 \AA$ at $H$ and $K(R \approx 330$ and 440). Individual exposure times were 120 seconds for the $J$ setting, 60 seconds for $H$ and 40 seconds for $K$, with total on-source exposure times of 48, 32, and 59 minutes at $J, H$, and $K$, respectively. The target was nodded along the slit by $60^{\prime \prime}$ in order to avoid scattered light from the very bright primary star. The spectrum of the target at $\lambda<1.2 \mu \mathrm{m}$ was still difficult to extract due to the light of the primary, and thus we do not use it. CGS4 has a calibration unit with lamps that provide accurate flat-fielding and wavelength calibration. The F6V star HD 615 was used as a calibrator to remove the effects of the terrestrial atmosphere, with $\mathrm{H}$ I recombination lines in its spectrum removed artificially prior to ratioing. The three separate spectra obtained for HD 3651B were merged together based on our UKIRT/UFTI photometry from the night before. After scaling, the 1.94-2.01 $\mu \mathrm{m}$ regions that overlapped in the $H$ and $K$-band spectra agreed to about $5 \times 10^{-18} \mathrm{~W} / \mathrm{m}^{2} / \mu \mathrm{m}$ (cf., the $K$ and $H$-band emission peaks of $2.1 \times 10^{-16}$ and $8.2 \times 10^{-16} \mathrm{~W} / \mathrm{m}^{2} / \mu \mathrm{m}$, respectively), thereby indicating the robustness of the merging process. 
We also obtained low-resolution $(R \approx 150)$ spectra of HD 3651B on 2006 September 13 UT from NASA's Infrared Telescope Facility (IRTF) located on Mauna Kea, Hawaii. Conditions were photometric with excellent seeing conditions, around 0.5" FWHM at $K$-band near zenith. We used the facility near-IR spectrograph Spex (Rayner et al. 1998) in prism mode, obtaining $0.8-2.5 \mu \mathrm{m}$ spectra in a single order. We used the $0.5^{\prime \prime}$ wide slit, oriented at the parallactic angle to minimize the effect of atmospheric dispersion. HD 3651B was nodded along the slit in an ABBA pattern, with individual exposure times of $200 \mathrm{sec}$, and observed over an airmass range of 1.00-1.08. The telescope was guided using images of the nearby star 2MASS J00391738+2115104 obtained with the near-IR slit-viewing camera. The total on-source exposure time was $73 \mathrm{~min}$. We observed the A0 V star HD 7215 contemporaneously with HD 3651B for flux and telluric calibration. All spectra were reduced using version 3.3 of the SpeXtool software package (Vacca et al. 2003; Cushing et al. 2004). The reduced IRTF/Spex spectrum is plotted in Figure1, in good agreement with the UKIRT/CGS4 spectrum. Also, the IRTF/Spex data provide the $\lambda \lesssim 1.2 \mu$ m region that was unobtainable with the UKIRT data.

\section{Results}

\subsection{Near-IR Spectral Types and Photometry}

Figure 1 presents our near-IR spectra of HD 3651B, showing the strong water and methane absorption bands that are the hallmarks of the T spectral class. Spectra of other late- $\mathrm{T}$ dwarfs are shown for comparison. We classified HD 3651B from the system of five spectral indices established by Burgasser et al. (2006b), as measured independently from our UKIRT/CGS4 and IRTF/Spex data (Table 2). The UKIRT/CGS4 data indicate a spectral type of T7.5, while the IRTF/Spex data indicate T8. (The difference does not arise from the differing spectral resolution of the two datasets, as we verified by smoothing the CGS4 data.) Figure 1 shows that the IRTF/Spex data slightly suggest a later type, based on the very slightly deeper $\mathrm{H}_{2} \mathrm{O}$ and $\mathrm{CH}_{4}$ absorption. However, in both datasets, HD 3651B does not appear as late as the T8 dwarf 2MASS 0415-0935 based on the depth of the $1.15 \mu \mathrm{m} \mathrm{H}_{2} \mathrm{O}$ absorption band. We also visually classified HD 3651B by comparing with UKIRT/CGS4 and IRTF/Spex spectra of late-T dwarfs classified by Burgasser et al., which have the same resolutions and instrumental setups as our data. For data from each instrument, the depth of the $\mathrm{H}_{2} \mathrm{O}$ and $\mathrm{CH}_{4}$ absorption bands were examined, normalizing the spectra of $\mathrm{HD}$ 3651B and the comparison objects to their peak fluxes in the $J, H$, and $K$-bands. This process confirmed that HD 3651B is later than T7 but earlier than T8. In fact, the depth of the absorption bands for HD $3651 \mathrm{~B}$ as judged by the indices and by eye are nearly identical to the T7.5 dwarf Gl 570D.

Therefore, we assign a spectral type of T7.5 for HD 3651B, with the nominal error of \pm 0.5 subclasses from the Burgasser et al. system. Our typing is in agreement with other results. Mugrauer et al. (2006) estimate T7-T8 based solely on the $H$-band absolute magnitude. Using independent sets of IRTF/Spex prism spectra, Luhman et al. (2006) determine T7.5 \pm 0.5 based 
on visual examination, and Burgasser (2006) find T8 \pm 0.5 based on spectral index measurements. With such a late-type spectrum, HD 3651B is unambiguously a substellar object.

Figure 2 shows our near-IR photometry of HD 3651B compared to other ultracool dwarfs; again, HD 3651B appears to be very similar to Gl 570D. Our UKIRT/UFTI $H$ and $K$-band photometry for HD 3651B agrees within the errors with photometry obtained by Luhman et al. (2006) using the IRTF/Spex slit-viewing camera. However, the $J$-band results differ by 0.15 mag, in that our UKIRT/UFTI measurement two months later is fainter. To explore this discrepancy, we used our single-order IRTF/Spex spectrum to synthesize the near-IR colors and found conflicting results: our Spex-synthesized $J-H$ agrees with our UKIRT/UFTI photometry but the synthesized $H-K$ is redder by 0.08 mag. It is likely that the discrepancy with the Luhman et al. $J$-band photometry is not significant at these levels. The IRTF/Spex slit-viewing camera is used primarily for acquisition and guiding, and it has not been rigorously tested for precision photometry, e.g. the linearity of the detector response has not been well-characterized (J. Rayner, priv. comm.). However, it is also possible that HD $3651 \mathrm{~B}$ is variable at the $\approx 10 \%$ level - further monitoring could be valuable.

Similarly, the $H-K$ discrepancy between our IRTF/Spex and UKIRT datasets is within the plausible errors of the overall flux calibration for the Spex data. Burgasser et al. (2006a) examined the consistency of broad-band photometric colors compared to colors synthesized from low-resolution IRTF/Spex spectra of 16 late-T dwarfs. They found typical deviations between the observed and synthesized colors of $5 \%$ or less, with a few sources having differences as large as $15 \%$.

\subsection{Age of the HD 3651AB System}

We consider several methods for establishing the age of the primary star HD 3651A. Age determination for main-sequence field stars is challenging and imperfect. For solar-type stars, methods for estimating ages largely rely on the increase in stellar rotation period as stars grow older. Stars spin down as they age because stellar winds carry away angular momentum; the increased rotation periods then lead to a decline in stellar activity due to the underlying stellar dynamo.

For solar-type stars, chromospheric activity as traced by CaII HK emission provides an age estimate. Donahue (1993, 1998) provide an age calibration for this index:

$$
\log (t)=10.725-1.334 R_{5}+0.4085 R_{5}^{2}-0.0522 R_{5}^{3}
$$

where $R_{5}=10^{5} R_{H K}^{\prime}$, valid for $\log \left(R_{H K}^{\prime}\right)=-4.25$ to -5.2 . Gray et al. (2003) measure $\log \left(R_{H K}^{\prime}{ }_{H}\right)=$ -5.09 for HD 3651A with an uncertainty of about 0.05 (R. Gray, priv. comm.) and describe it as an inactive star. Wright et al. (2004) find $\log \left(R_{H K}^{\prime}\right)=-5.02$, with a full range of about $10 \%$ over 7 years of measurements. (Duncan et al. 1991 report a $40 \%$ change in CaII HK emission over 16 years of monitoring.) These measurements lead to an age estimate of about 5-9 Gyr. An error 
estimate is not available for this technique. However, out of a sample of 21 binaries studied by Donahue (1998), most had age estimates differing by $\lesssim 2$ Gyr.

X-ray emission of solar-type stars also declines with age. Hempelmann et al. (1995) measure $L_{X} / L_{b o l}=-5.69$ for HD 3651A with a 30\% uncertainty. Gaidos (1998) provides an age calibration based on scaling relations for stellar activity:

$$
\log \left(L_{X} / L_{b o l}\right)=-6.38-2.6 \alpha \log \left(t_{9} / 4.6\right)+\log \left[1+0.4\left(1-t_{9} / 4.6\right)\right]
$$

where $t_{9}$ is the age in Gyr and $\alpha$ is the coefficient that relates rotation period to stellar age, either $\alpha=0.5$ (Skumanich 1972) or $\alpha=1 / e$ (Walter \& Berry 1991). Following Wilson et al. (2001), we adopt the zeropoint of -6.38 based on the X-ray luminosity of the Sun from Maggio et al. (1987). Including the uncertainties, we estimate an age of 0.9-2.2 Gyr for HD 3651A. As a point of reference, the X-ray luminosity of HD 3651A is $\log \left(L_{X}\right)=27.6$ (Hempelmann et al. 1995). This is about 6 times fainter than Hyades stars of similar spectral type (Stern et al. 1995). Preibisch \& Feigelson (2005) estimate $L_{X} \propto t^{-0.77}$ for solar-type stars, implying that HD 3651A is about $4 \times$ older than the Hyades (650 Myr) or around 3 Gyr.

Due to angular momentum carried away by stellar winds, the rotation periods of solar-type stars increase as they age, believed to follow a power-law relation of $P_{\text {rot }} \sim t^{\alpha}$ (e.g. Skumanich 1972), where $\alpha$ is the same as in Equation 2. Baliunas et al. (1983) report a period of $48 \mathrm{~d}$ for HD 3651A's chromospheric activity, which we adopt as the stellar rotation period. Using the Sun as a reference point $\left(P_{\text {rot }}=26 \mathrm{~d}\right.$ and $t=4.6 \mathrm{Gyr}$ ), the scaling relation gives an implausibly large age of 16-24 Gyr. Lachaume et al. (1999) have provided an age calibration for main-sequence stars based on a sample from the Hipparcos catalog:

$$
\log \left(t_{9}\right)=2.667 \log (P)-0.944(B-V)-0.309[\mathrm{Fe} / \mathrm{H}]+6.530 \text {. }
$$

where $t_{9}$ is the age in Gyr and $P$ is the period in days. With $B-V=0.85$ mag (Perrvman et al. 1997) and $[\mathrm{Fe} / \mathrm{H}]=0.09-0.16$ (Grav et al. 2003; Santos et al. 2004; Valenti \& Fischer 2005), this gives an age of 15 Gyr. Lachaume et al. (1999) caution that their relation is less accurate for stars older than 3 Gyr.

Finally, from high resolution spectroscopic analysis combined with bolometric magnitudes and theoretical stellar evolutionary isochrones, Valenti \& Fischer (2005) derive an age estimate of 8.2 Gyr with a possible range of 3-12 Gyr, and Takeda et al. (2006) infer a minimum age of 11.8 Gyr. The quoted age range from Valenti \& Fischer spans the aforementioned activity-based estimates from the X-ray data $(\approx 1-3 \mathrm{Gyr})$ and CaII HK data $(\approx 5-9$ Gyr), and thus we adopt an age of 3-12 for HD 3651A, with a geometric mean of 6 Gyr. This old age is supported by the star's slow rotation and the Takeda et al. estimate. 


\subsection{Luminosity, Mass, and Effective Temperature}

To detemine the bolometric luminosity of HD $3651 \mathrm{~B}$, we use a $K$-band bolometric correction $\left(B C_{K}\right)$ of $2.07 \pm 0.13 \mathrm{mag}$, based on the Golimowski et al. (2004) polynomial relation of $B C_{K}$ versus near-IR spectral type. This gives $\log \left(L_{\mathrm{bol}} / L_{\odot}\right)=-5.58 \pm 0.05$, with the uncertainty coming from the quadrature sum of the uncertainties in the $K$-band absolute magnitude, the distance to the system, the $B C_{K}$ due to the spectral typing uncertainty, and the scatter about the fitted polynomial relation. HD $3651 \mathrm{~B}$ has the second smallest $L_{\text {bol }}$ measured among all brown dwarfs with trigonometric distances, comparable to that of the T7.5 dwarf Gl 570D and exceeded only by the T8 dwarf 2MASS 0415-0935, which have $\log \left(L_{\mathrm{bol}} / L_{\odot}\right)=-5.53 \pm 0.05$ and $-5.73 \pm 0.07$, respectively (Golimowski et al. 2004) 1

Instead of the $B C_{K}$ from the polynomial relation as a function of spectral type, we could also have used the individual $B C_{K}$ values determined for the four late-T dwarfs in the Golimowski et al. (2004) sample: 2MASS 0727+1710 (T7; $\left.B C_{K}=2.24 \pm 0.13 \mathrm{mag}\right)$, Gl 570D (T7.5; $\left.1.90 \pm 0.13 \mathrm{mag}\right)$; 2MASS 1217-0311 (T7.5; 2.28 $\pm 0.13 \mathrm{mag}$ ); and 2MASS 0415-0935 (T8; $2.03 \pm 0.13 \mathrm{mag}$ ), where the spectral types are those assigned by Burgasser et al. (2006b). The average of the two T7.5 dwarfs is $2.09 \mathrm{mag}$, consistent with our adopted value. If we adopt the Gl 570D value based on its close spectral resemblance to HD $3651 \mathrm{~B}$, we would obtain an $L_{\text {bol }}$ value 0.08 dex brighter, which would not have a significant impact on our conclusions. Finally, Luhman et al. (2006) used nearIR spectra, Spitzer/IRAC thermal IR (3.2-9.2 $\mu \mathrm{m})$ photometry, and an assumed long wavelength Rayleigh-Jeans distribution to derive a bolometric luminosity of $\log \left(L_{\mathrm{bol}} / L_{\odot}\right)=-5.60 \pm 0.05$, in excellent agreement with our assessment.

To estimate the $T_{\text {eff }}$ and mass of HD 3651B, we use the observational constraints of the derived $L_{\text {bol }}$ and estimated age of $6_{-3}^{+6}$ Gyr combined with the solar-metallicity evolutionary models of Burrows et al. (1997). For a nominal age of $6 \mathrm{Gyr}$, we find an effective temperature of $810 \mathrm{~K}$ and a mass of $56 M_{\mathrm{Jup}}$. The ranges of these values are $780-840 \mathrm{~K}$ and masses of $40-72 M_{\mathrm{Jup}}$, with younger assumed ages leading to cooler temperatures and lower masses. The resulting derived properties are given in Table 12

\footnotetext{
${ }^{1}$ Analysis of the near-IR spectra of the T8 dwarf 2MASS 0939-2448 and the T7.5 dwarf 2MASS 1114-2618 by Burgasser et al. (2006a) suggest that these two objects may be even cooler and lower luminosity than 2MASS 0415-0935, though they do not yet have parallaxes measurements.

${ }^{2}$ In principle, one should use evolutionary models computed for the same metallicity as the parent stars. However, the slightly super-solar metallicity of HD 3631A (discussed in $\S 4$ ) should not lead to a significant difference in the derived properties. Tables 2 and 3 of Saumon et al. (2006) show that for late-T dwarfs, changing the metallicity from 0.0 dex to 0.3 dex changes the model-derived mass by $\lesssim 5 \%, T_{\text {eff }}$ by $\lesssim 1 \%$, and $\log (g)$ by $\lesssim 0.05$ dex for a given age.
} 


\section{Discussion}

\subsection{Relative Ages, Masses and Temperatures of HD 3651B and Gl 570D}

HD 3651B is remarkably similar to the T7.5 dwarf Gl 570D, a wide-separation companion to the triple system Gl 570ABC (Burgasser et al. 2000; Geballe et al. 2001), separated by 1525 AU from the $\mathrm{K} 4 \mathrm{~V}$ primary star. These two brown dwarfs have very comparable properties, namely their nearIR spectra (both spectral type T7.5), JHK absolute magnitudes (HD 3651B is $\lesssim 0.1$ mag fainter), and $J H K$ colors (HD 3651B is redder by $\lesssim 0.15 \mathrm{mag}$ ). Their primary stars both have about solar metallicity, $[\mathrm{Fe} / \mathrm{H}]=0.01-0.10$ for Gl 570A (Cayrel de Strobel et al. 1997; Feltzing \& Gustafsson 1998; Santos et al. 2005; Valenti \& Fischer 2005) and $[\mathrm{Fe} / \mathrm{H}]=0.09-0.16$ for HD 3651A (Grav et al. 2003; Santos et al. 2004; Valenti \& Fischer 2005). Hence, we would expect that differences between these two brown dwarfs arise primarily from their differing masses and ages, whose observational manifestation is surface gravity. Geballe et al. (2001) estimated an age of 2-5 Gyr for Gl 570A, younger than our estimate of 3-12 Gyr for HD 3651A, but the two estimates derive from different methods. Here we re-examine the relative ages of these two primary stars and in the next section consider the implications in interpreting the spectra of their late- $T$ dwarf companions.

Gl 570A is more chromospherically active as judged by its $\log \left(R_{H K}^{\prime}\right)$ values -4.49 and -4.75 (Henry et al. 1996; Soderblom et al. 1991), compared to $\log \left(R_{H K}^{\prime}\right) \approx-5.05$ for HD 3651A (§ 3.2). Equation 1 gives corresponding ages of 0.8 and 2.2 Gyr for Gl $570 \mathrm{~A}$, compared to $\approx 7$ Gyr for HD 3651A 3 Geballe et al. (2001) suggested that the young age inferred from the CaII HK data may be due to the fact that Gl 570A was observed during a period of enhanced activity. As a point of reference, the Sun varies from $\log \left(R_{H K}^{\prime}\right)=-5.10$ to -4.75 , which would lead to chromospherically inferred ages of 3-8 Gyr (e.g. Baliunas et al. 1998). Assuming the CaII HK variability of Gl 570A and HD 3651A is comparable to the solar cycle, the two stars would have to had been observed at nearly the opposite extremes of their activity cycles to still have the same age. We discount this possibility and conclude that the chromospheric data indicate that Gl 570A is younger than HD 3651A.

Similarly, the other age indicators we have considered support a younger age for Gl 570A compared to HD 3651A. Based on $\log \left(L_{X}\right)=27.7$ from Schmitt \& Liefke (2004) and bolometric corrections from Kenyon \& Hartmann (1995), we find Gl 570A has $\log \left(L_{X} / L_{\mathrm{bol}}\right)=-5.27$. Using Equation 2, this gives an age of 0.4-0.8 Gyr, compared to 1-3 Gyr inferred for HD 3651A from the same approach. As pointed out by Geballe et al. (2001), Gl 570A has a lower X-ray luminosity compared Hyades stars of similar spectral type, setting a lower age limit of $650 \mathrm{Myr}$. Also, Gl 570A has a somewhat shorter rotation period of 40 days, compared to 48 days for HD 3651A, implying a $\approx 50-60 \%$ younger age for Gl $570 \mathrm{~A}$ based on Equation 3 (§3.2). Finally, isochrone analyses

3 Strassmeier et al. (2000) report $\log \left(R_{H K}^{\prime}\right)=-4.21$ for Gl $570 \mathrm{~A}$, which is higher level of activity than covered by the Donahue (1998) calibration, suggesting an age of $\lesssim 10$ Myr. However, the absence of Li I $6708 \AA$ absorption indicates that Gl 570A is at least a zero-age main sequence star (Rocha-Pinto et al. 2002). 
for Gl 570A by Valenti \& Fischer (2005) and Takeda et al. (2006) give ages of $3.3_{-3.1}^{+8.3}$ Gyr and $<0.6$ Gyr, respectively.

Table 3 summarizes the age estimates for HD 3651A and Gl 570A. While there is considerable scatter in the absolute ages, the data all suggest that Gl $570 \mathrm{~A}$ is $\approx 3 \times$ younger than HD $3651 \mathrm{~A}$. In the analysis that follows, we adopt a conservative age range of $1-5$ Gyr for Gl 570D, with a geometric mean of 2 Gyr. The upper limit of 5 Gyr is the same as that of Geballe et al. (2001) and derives from the scatter of the Donahue (1998) calibration sample. Our adopted lower age limit of 1 Gyr is younger than the 2 Gyr used by Burgasser et al. (2000) and Geballe et al. (2001). Their value is based on the kinematical age of the system as derived from its $(U, V, W)$ space motion and the absence of $\mathrm{H} \alpha$ emission from the Gl 570BC M-type binary. The former is a statistical measure and therefore is not a very strong constraint for an individual star. The latter is also not a strong constraint, since the age dependence of $\mathrm{H} \alpha$ emission for field $\mathrm{M}$ dwarfs is also largely based on kinematic analyses (Hawlev et al. 1996). The $\approx 1$ Gyr lower age limit discussed here based on stellar activity indicators for the K-type primary Gl 570A is a more conservative estimate, especially since these indicators are calibrated with data from open clusters 4

Assuming that the brown dwarfs are coeval with their primary stars, Figure 3 illustrates the model-derived masses, surface gravities, and effective temperatures for HD 3651B and Gl 570D, based on the Burrows et al. (1997) evolutionary models and the observational constraints in Table 1 . The $L_{\text {bol }}$ measurements more strongly constrain $T_{\text {eff }}$, while the age of the primary star sets $\log (g)$. For Gl 570D and a nominal age of 2 Gyr, the bolometric luminosity of $10^{-5.53 \pm 0.05} L_{\odot}$ (Golimowski et al. 2004) gives an effective temperature of $780 \mathrm{~K}$ and a mass of $33 M_{\text {Jup }}$, with ranges of $750-825 \mathrm{~K}$ and $24-51 M_{\text {Jup }}$ for ages of $1-5$ Gyr. (Note that the apparent overlap of the uncertainties in the Figure 3 is misleading, since the uncertainties represent the plausible spread in the absolute ages. The relative ages of the primary stars are known to better accuracy, as just discussed.)

\subsection{Surface Gravity and Metallicity Effects in Late-T Dwarf Spectra}

Given the age and metallicity determinations for the primary stars, HD 3651B and Gl 570D provide two benchmarks for examining the differential effects of surface gravity and metallicity on the spectra of late-T dwarfs. Figure 3 shows that the older age of HD 3651B leads to a $\approx 0.3$ dex higher inferred surface gravity compared to Gl 570D, with nearly the same $T_{\text {eff }}$ for the two objects. For late- $\mathrm{T}$ dwarfs, this difference would be most prominently manifested in the $K$-band emission, which is heavily influenced by opacity from collision-induced $\mathrm{H}_{2}$ absorption (e.g. Linsky 1969). Higher surface gravity objects will have higher photospheric pressures, and thus the $\mathrm{H}_{2}$ opacity

\footnotetext{
${ }^{4}$ Saumon et al. (2006) suggest an age range of 3-5 Gyr for the Gl 570ABCD system, based on modeling the optical to mid-IR spectrum of the T-dwarf companion Gl 570D.
} 
will be stronger and the $K$-band flux more heavily depressed. Indeed, variations in the near-IR properties of late- $T$ dwarfs are typically attributed to variations in surface gravity (e.g. Knapp et al. 2004; Burgasser et al. 2006a; Burrows et al. 2006), which translates into variations in mass because the radii of field brown dwarfs are very similar.

However, the inferred surface gravity difference does not seem to be in accord with the appearance of the near-IR spectra of these two objects. Since HD 3651B and Gl 570D have almost identical metallicities $(\Delta[\mathrm{Fe} / \mathrm{H}] \approx 0.1 \mathrm{dex})$ and effective temperatures $\left(\Delta T_{\text {eff }} \approx 30 \mathrm{~K}\right)$, the higher surface gravity of HD 3651B should lead to bluer near-infrared colors compared to Gl 570D due to stronger $\mathrm{H}_{2}$ opacity. But instead the observed colors of HD $3651 \mathrm{~B}$ are redder by $0.12 \pm 0.08$ and $0.15 \pm 0.08 \mathrm{mag}$ at $H-K$ and $J-K$, respectively. Thus, there appears to be a discrepancy between the expected behavior of $\mathrm{H}_{2}$ opacity and the actual HD 3651B/Gl 570D comparison. Similarly, Burgasser et al. (2006a) use the pressure sensitivity of $\mathrm{H}_{2}$ opacity to constrain the surface gravity of $\mathrm{T}$ dwarfs through spectral indices that ratios the $K$ and $H$-band peak fluxes. Their Figure 5 implies that increasing gravity by $0.3 \mathrm{dex}$ at $T_{\text {eff }} \approx 800 \mathrm{~K}$ should produce a decrease in the $K / H$ ratios of $\approx 20 \%$ - instead we see an increase of $17 \pm 8 \%$ (Table 4 ). In short, comparing the near-IR colors and spectra of HD 3651B and Gl 570D suggest that HD 3651B has a lower surface gravity and thus a younger age, in contradiction to the relative ages inferred for their primary stars in $\S 4.1$.

We suggest that this apparent discrepancy arises from the small metallicity difference $(\approx 0.1$ dex $)$ between the two systems. Collision-induced $\mathrm{H}_{2}$ opacity is also expected to be affected by metallicity variations (Borysow et al. 1997). Higher metallicities lead to more opaque atmospheres; therefore, the photosphere resides at lower pressures and collision-induced $\mathrm{H}_{2}$ opacity is reduced. Models of $\mathrm{T}$ dwarf spectra by Burrows et al. (2006) as a function of metallicity $([\mathrm{Fe} / \mathrm{H}]=-0.5$ to +0.5$)$ and surface gravity $(\log (g)=4.5$ to 5.5$)$ show that both variables can have strong effects on the emergent near-IR SED. For instance, Figure 20 of Burrows et al. shows that at fixed $T_{\text {eff }}$, the $J-K$ model colors vary greatly with metallicity and surface gravity and that the two quantities act in opposite senses, as expected: the higher surface gravities that produce bluer near-IR colors can be counteracted by higher metallicities leading to redder colors. Likewise, models by M. Marley et al. (in prep.) suggest that the $\approx 0.1$ dex metallicity difference between HD 3651B and Gl 570D could counteract and even outweigh the effect of the 0.3 dex difference in $\log (g)$ on their relative $K$-band fluxes.

To quantify the sensitivity of near-IR spectra to changes in metallicity and surface gravity, we examine the condensate-free atmospheric models from the Tucson group, as described in Burrows et al. (2002) and Burrows et al. (2006). Burgasser et al. (2006a) define a set of 5 spectral indices to characterize the emission peaks and $\mathrm{H}_{2} \mathrm{O}$ absorption in late- $\mathrm{T}$ dwarf spectra; our measurements for HD 3651B and Gl 570D are given in Table 4. For a particular spectral index, one can envision that its model-predicted values constitute a 3-dimensional surface in the parameter space of $\left\{T_{\text {eff }}, \log (g), Z\right\}$. We compute the local slope of the surface to quantify how the index varies with effective temperature, surface gravity, and metallicity about nominal reference values 
of $T_{\text {eff }}=800 \mathrm{~K}, \log (g)=5.0$ and $Z / Z_{\odot}=1.0$. In practice, the indices do not vary linearly, and thus we compute the changes both for decreasing and increasing values of $T_{\text {eff }}, \log (g)$, and $Z$ about the reference values. For example, the $\mathrm{H}_{2} \mathrm{O}-J$ and $\mathrm{H}_{2} \mathrm{O}-H$ indices from the models change strongly from $Z / Z_{\odot}=0.3$ to 1.0 but not very much for $Z / Z_{\odot}=1.0$ to 3.0 .

Figure 4 provides a pictoral representation of the metallicity and surface gravity sensitivities for $T_{\text {eff }}=700$ to $900 \mathrm{~K}$. The results for the $800 \mathrm{~K}$ models are also listed in Table 5. The models predict that indices involving the $K$-band flux, namely $K / H$ and $K / J$, are strongly sensitive to both metallicity and surface gravity, and less sensitive to $T_{\text {eff }}$. Table 5 indicates that a 0.1 dex greater metallicity for HD 3651B compared to Gl 570D will produce a $2.41 \times 0.1=24 \%$ increase in $K / H$, compared to the $-0.58 \times 0.3=17 \%$ decrease due to a 0.3 dex higher surface gravity, consistent with our hypothesis that that the enhanced $K$-band flux of HD 3651B is due to its higher metallicity, despite its higher surface gravity 5 For comparison, Table 5 also indicates that a $30 \mathrm{~K}$ difference in $T_{\text {eff }}$ leads to only a $0.21 \times(30 / 100)=6 \%$ change in $K / H$.

But are the model predictions correct? A comparison of HD 3651B and Gl 570D allows, for the first time, an empirical test of the differential effects of $\log (g)$ and metallicity on late-T dwarf spectra. Based on the properties compiled in Table 1, we adopt a difference of $0.25 \pm 0.05$ dex in surface gravity, $0.10 \pm 0.05$ dex in metallicity, and $20 \pm 10 \mathrm{~K}$ in temperature between these two objects, with all the quantities being larger for HD 3651B. Table 4 summarizes the results. The model predictions for $K / H$ agree best with the observations given the uncertainties, showing the index increases for HD 3651B compared to Gl 570D. The other model results do not agree so well with the observations, with those involving the $J$-band flux being particularly poor $(Y / J$ and $K / J$ ), and is likely affected by missing $\mathrm{CH}_{4}$ and $\mathrm{NH}_{3}$ opacities in the $Y J H$-bands (e.g., Figure 15 of Burrows et al. 2001). The $Y$-band fluxes are also subject to uncertainties in the far-red opacity wings of the K I $0.77 \mu \mathrm{m}$ resonance doublet; the Tucson models employed here are based on the modified Lorentzian profiles from Burrows \& Sharp (1999), as opposed to more recent calculations by Burrows \& Volobuvev (2003).

Burgasser et al. (2006a) have used the same Burrows et al. models, largely restricted to solar metallicity, to determine $T_{\text {eff }}$ and $\log (g)$ for late-T dwarfs from near-IR spectra and, combined with evolutionary models, to estimate ages and masses. In principle, such a method could be quite valuable, as the key physical parameters of the coldest known brown dwarfs could be extracted solely from low-resolution near-IR spectra. They calibrate their method by scaling the models to agree with the $T_{\text {eff }}$ and $\log (g)$ determined for Gl 570D by Geballe et al. (2001). While such a calibration is necessary, it is not complete, since it only requires the models to agree with a single object. HD 3651B now provides second calibration point, and our differential comparison

\footnotetext{
${ }^{5}$ The difficulties of disentangling surface gravity and metallicity effects are also demonstrated by Liebert \& Burgasser (2006). Their analysis of the unusual strong H $\alpha$-emitting T6.5 dwarf 2MASS $1237+6526$ highlights the degeneracy betwen inferring subsolar metallicity and reduced surface gravity from low-resolution near-IR spectra.
} 
indicates that (1) non-solar metallicities must be considered to determine the correct $\log (g)$ (and thus the mass), and (2) model predictions for indices involving the $J$-band flux may not be correct. Improvements in the models and identification of more calibration objects will be needed to fully exploit the potential of the Burgasser et al. (2006a) approach 6

Our comparison here of the near-IR colors and low-resolution spectra of HD 3651B and Gl 570D indicates that metallicity effects are significant in late- $\mathrm{T}$ dwarf spectra, perhaps more important than previously appreciated. If this indeed is the case, then disentangling the effects of gravity and metallicity variations among the field population may be quite challenging. In a similar vein, analyses that consider only solar metallicity models (e.g. Knapp et al. 2004; Burgasser et al. 2006a) will naturally infer an inflated spread in $\log (g)$ for the late-T dwarfs compared to the true spread. Since field brown dwarfs have very similar radii, errors in $\log (g)$ determinations translate almost directly into errors in the masses.

We provide here a simple estimate of the relative importance of metallicity versus surface gravity variations. At $T_{\text {eff }}=700-900 \mathrm{~K}$, objects with ages of 1-10 Gyr correspond to masses of 20$65 M_{\text {Jup }}$, based on Burrows et al. (1997) models. Given the nearly constant radii of old substellar objects, this age spread amounts to about a 0.5 dex variation in surface gravity. The metallicity spread of the field population is about the same; for instance, Rocha-Pinto \& Maciel (1996) find that $\approx 90 \%$ of the $\mathrm{G}$ dwarfs in the solar neighborhood cover a metallicity spread of $\approx 0.6$ dex. For comparison, Figure 4 and Table 5 indicate that the $K / H$ index changes by $100-240 \%$ per dex of $\log (Z)$ change and by $60-100 \%$ per $\operatorname{dex}$ of $\log (g)$ change, i.e., that $K / H$ is about twice as sensitive to metallicity as compared to surface gravity. Given the similar spread in $\log (g)$ and $\log (Z)$ in the field population, the models therefore predict that metallicity variations will have a larger effect than surface gravity variations on the spectral properties of the late- $\mathrm{T}$ dwarfs 7

\section{Conclusions}

We have obtained near-IR photometry and spectroscopy of HD 3651B, the wide-separation, low-luminosity companion to the nearby K0V star HD 3651A identified by Mugrauer et al. (2006) and Luhman et al. (2006). We find a spectral type of T7.5 \pm 0.5 for HD 3651B. This makes it the 6th $\mathrm{T}$ dwarf with a spectral type later than $\mathrm{T} 7$ and only the 4 th with a trigonometric parallax. It is also the 6th T dwarf found as a companion, the other ones being Gl 229B (T7p; Nakajima et al.

\footnotetext{
Eurgasser (2006) have applied this method to near-IR spectra of HD 3651B. Similar to us, they find that accounting for metallicity is important in extracting the physical parameters. However, they infer a $\log (g)$ and age similar to Gl 570D, even after accounting for the super-solar metallicity of HD 3651B. This is in discord with our conclusion that the HD $3651 \mathrm{~B}$ is $\approx 3 \times$ older than Gl $570 \mathrm{D}$ and thus has a higher surface gravity.

${ }^{7}$ In fact, the empirical comparison of HD 3651B to Gl 570D suggests an even larger effect due to metallicity than the models do. Table 4 shows that $K / H$ changes by almost twice as much between these two objects as the models predict.
} 
1995), Gl 570D (T7.5; Burgasser et al. 2000), $\epsilon$ Ind Bab (T1+T6 binary; McCaughrean et al. 2004), and SCR 1845-6357B ( $\approx$ T5.5; Biller et al. 2006). We find that $T_{\text {eff }}$ and $L_{\text {bol }}$ for HD $3651 \mathrm{~B}$ are among the lowest determined for any brown dwarf with a trigonometric parallax, comparable to those of the T7.5 dwarf Gl 570D and exceeded only by the T8 dwarf 2MASS 0415-0935.

HD 3651B appears to be very similar to Gl 570D, the other known late-T dwarf companion to a $\mathrm{K}$ dwarf. Given their similar temperatures $\left(\Delta T_{\text {eff }} \approx 30 \mathrm{~K}\right)$ and the similar metallicities of their host stars $(\Delta[\mathrm{Fe} / \mathrm{H}] \approx 0.1$ dex $)$, a comparison of HD 3651B to Gl 570D offers a probe of surface gravity effects in ultracool atmospheres. While absolute age determinations are difficult for field dwarfs, several methods indicate that HD 3651A (3-12 Gyr) is notably $(\approx 3 \times)$ older than Gl 570A (1-5 Gyr), and consequently evolutionary models show that HD 3651B has a $\approx 0.3$ dex higher surface gravity than Gl 570D. However, the near-IR colors and spectra of HD 3651B compared to Gl 570D appear to be at variance with the relative ages of the host stars, with the $K$-band fluxes indicating a lower surface gravity, and thus younger age, for HD 3651B. Hence, at face value the relative ages these two brown dwarfs derived from activity-based indicators for primary stars and from the interpretation of late- $\mathrm{T}$ dwarf spectra appear to be in discord.

We suggest that this discrepancy arises from the small $(\approx 0.1 \mathrm{dex})$ metallicity difference between the two objects, reflecting the metallicity sensitivity of the collision-induced $\mathrm{H}_{2}$ opacity that shapes the $K$-band flux. Given the physical properties established from their host stars, HD $3651 \mathrm{~B}$ and Gl 570D offer a differential test of gravity and metallicity effects in late-T dwarf spectra. We find that the the condensate-free models of Burrows et al. (2006) are in fair agreement with the observed change in $K / H$ index but other indices, especially those involving the $J$-band flux, do not match the observations very well. Simple estimates based on the plausible metallicity and gravity spread among the field population suggest that metallicity may be more important than surface gravity in producing spectral variations among late-T dwarfs. Hence, theoretical atmospheres that include non-solar metallicities will be valuable for interpreting these ultracool objects.

Further observations of the HD 3651AB and Gl 570ABCD system will clarify the relative roles of metallicity and surface gravity in late- $T$ dwarf spectra. More accurate constraints on the ages of the primary stars would better constrain the surface gravities (e.g., Figure 3). Higher resolution near-IR spectra, e.g., to resolve the $J$-band K I $1.25 \mu \mathrm{m}$ doublet, might probe gravity and metallicity diagnostics, as could spectra at far-red optical wavelengths (e.g. Burrows et al. 2002; Burgasser et al. 2003). Discovery of more late-T dwarfs as stellar companions and/or members of nearby star clusters will provide more benchmarks for comparative study over a range of effective temperatures, surface gravities, and metallicities.

Finally, confirmation of the substellar status of HD 3651B demonstrates that wide-separation brown dwarf companions and giant planets can co-exist around the same star. While brown dwarf companions have been resolved around other stars (e.g. Becklin \& Zuckerman 1988; Nakaiima et al. 1995; Rebolo et al. 1998; Kirkpatrick et al. 2001; Liu et al. 2002; Metchev \& Hillenbrand 2004), and there are also several systems with both brown dwarf and planetary companions found by 
radial velocity searches (e.g. Marcy et al. 2001; Udry et al. 2002), the HD 3651ABb system is the first with a radial velocity planet and a wide-separation (resolved) brown dwarf. The upcoming Pan-STARRS project (Kaiser et al. 2002) will monitor the entire sky observable from Hawai'i and produce sensitive multi-band optical photometry with high astrometric precision. This should be promising means to identify more wide-separation substellar companions to stars by their common parallax and proper motion, including stars being monitored by high precision radial velocity surveys. Therefore, as our census of the solar neighborhood continues to become more complete, so will our appreciation for the diversity of planetary systems.

It is a pleasure to thank Paul Hirst, Tim Carroll, John Rayner, Bill Golisch, Alan Tokunaga, and the staff of UKIRT and IRTF for their assistance with the observations. We thank Adam Burrows, Kevin Luhman, and Adam Burgasser for providing digital versions of published results, and thank John Rayner and Michael Cushing for assistance with SpeXtool. We are grateful to our anonymous referee, Adam Burrows, and Katelyn Allers for useful comments. Our research has benefitted from the 2MASS data products; NASA's Astrophysical Data System; and the SIMBAD database operated at CDS, Strasbourg, France. MCL acknowledges support for this work from NSF grants AST-0407441 and AST-0507833 and an Alfred P. Sloan Research Fellowship. SKL is supported by the Gemini Observatory, which is operated by the Association of Universities for Research in Astronomy, Inc., on behalf of the international Gemini partnership of Argentina, Australia, Brazil, Canada, Chile, the United Kingdom, and the United States of America. The United Kingdom Infrared Telescope is operated by the Joint Astronomy Centre on behalf of the U.K. Particle Physics and Astronomy Research Council. Finally, the authors wish to recognize and acknowledge the very significant cultural role and reverence that the summit of Mauna Kea has always had within the indigenous Hawaiian community. We are most fortunate to have the opportunity to conduct observations from this mountain.

\section{REFERENCES}

Baliunas, S. L., Donahue, R. A., Soon, W., \& Henry, G. W. 1998, in ASP Conf. Ser. 154: Cool Stars, Stellar Systems, and the Sun, ed. R. A. Donahue \& J. A. Bookbinder, 153

Baliunas, S. L. et al. 1983, ApJ, 275, 752

Becklin, E. E. \& Zuckerman, B. 1988, Nature, 336, 656

Biller, B. A., Kasper, M., Close, L. M., Brandner, W., \& Kellner, S. 2006, ApJ, 641, L141

Borysow, A., Jorgensen, U. G., \& Zheng, C. 1997, A\&A, 324, 185

Burgasser, A. J. 2006, ApJ, submitted (astro-ph/0609556)

Burgasser, A. J., Burrows, A., \& Kirkpatrick, J. D. 2006a, ApJ, 639, 1095 
Burgasser, A. J., Geballe, T. R., Leggett, S. K., Kirkpatrick, J. D., \& Golimowski, D. A. 2006b, ApJ, 637

Burgasser, A. J., Kirkpatrick, J. D., Liebert, J., \& Burrows, A. 2003, ApJ, 594, 510

Burgasser, A. J. et al. 1999, ApJ, 522, L65

-. 2000, ApJ, 531, L57

Burrows, A., Burgasser, A. J., Kirkpatrick, J. D., Liebert, J., Milsom, J. A., Sudarsky, D., \& Hubeny, I. 2002, ApJ, 573, 394

Burrows, A., Hubbard, W. B., Lunine, J. I., \& Liebert, J. 2001, Reviews of Modern Physics, 73, 719

Burrows, A., Marley, M., Hubbard, W. B., Lunine, J. I., Guillot, T., Saumon, D., Freedman, R., Sudarsky, D., \& Sharp, C. 1997, ApJ, 491, 856

Burrows, A. \& Sharp, C. M. 1999, ApJ, 512, 843

Burrows, A., Sudarsky, D., \& Hubeny, I. 2006, ApJ, 640, 1063

Burrows, A. \& Volobuyev, M. 2003, ApJ, 583, 985

Carson, J. C., Eikenberry, S. S., Smith, J. J., \& Cordes, J. M. 2006, AJ, 132, 1146

Cayrel de Strobel, G., Soubiran, C., Friel, E. D., Ralite, N., \& Francois, P. 1997, A\&AS, 124, 299

Chiu, K. et al. 2006, AJ, 131, 2722

Cushing, M. C., Vacca, W. D., \& Rayner, J. T. 2004, PASP, 116, 362

Dahn, C. C. et al. 2002, AJ, 124, 1170

Donahue, R. A. 1993, PhD thesis, New Mexico State University

Donahue, R. A. 1998, in ASP Conf. Ser. 154: Cool Stars, Stellar Systems, and the Sun, Vol. 10, 1235

Duncan, D. K. et al. 1991, ApJS, 76, 383

Feltzing, S. \& Gustafsson, B. 1998, A\&AS, 129, 237

Fischer, D. A., Butler, R. P., Marcy, G. W., Vogt, S. S., \& Henry, G. W. 2003, ApJ, 590, 1081

Gaidos, E. J. 1998, PASP, 110, 1259

Geballe, T. et al. 2002, ApJ, 564, 466 
Geballe, T. R., Saumon, D., Leggett, S. K., Knapp, G. R., Marley, M. S., \& Lodders, K. 2001, ApJ, 556, 373

Golimowski, D. A. et al. 2004, AJ, 127, 3516

Gray, R. O., Corbally, C. J., Garrison, R. F., McFadden, M. T., \& Robinson, P. E. 2003, AJ, 126, 2048

Hawley, S. L., Gizis, J. E., \& Reid, I. N. 1996, AJ, 112, 2799

Hempelmann, A., Schmitt, J. H. M. M., Schultz, M., Ruediger, G., \& Stepien, K. 1995, A\&A, 294, 515

Henry, T. J., Soderblom, D. R., Donahue, R. A., \& Baliunas, S. L. 1996, AJ, 111, 439

Kaiser, N. et al. 2002, in Survey and Other Telescope Technologies and Discoveries. Edited by Tyson, J. Anthony; Wolff, Sidney. Proc. of the SPIE, Volume 4836, pp. 154-164 (2002)., $154-164$

Kenyon, S. J. \& Hartmann, L. 1995, ApJS, 101, 117

Kirkpatrick, J. D. 2005, ARA\&A, 43, 195

Kirkpatrick, J. D., Dahn, C. C., Monet, D. G., Reid, I. N., Gizis, J. E., Liebert, J., \& Burgasser, A. J. 2001, AJ, 121, 3235

Knapp, G. R. et al. 2004, AJ, 127, 3553

Lachaume, R., Dominik, C., Lanz, T., \& Habing, H. J. 1999, A\&A, 348, 897

Leggett, S. K. et al. 2000, ApJ, 536, L35

-. 2006, MNRAS, 1209

Liebert, J. \& Burgasser, A. J. 2006, ApJ, in press (astro-ph/0609793

Linsky, J. L. 1969, ApJ, 156, 989

Liu, M. C., Fischer, D. A., Graham, J. R., Lloyd, J. P., Marcy, G. W., \& Butler, R. P. 2002, ApJ, 571,519

Liu, M. C., Leggett, S. K., Golimowski, D. A., Chiu, K., Fan, X., Geballe, T. R., Schneider, D. P., \& Brinkmann, J. 2006, ApJ, 647, 1393

Lloyd, J. P., Liu, M. C., Graham, J. R., et al. 2004, in IAU Symposium, ed. A. Penny, 462

Luhman, K. L. et al. 2006, ApJ, in press (astro-ph/0609464) 
Maggio, A., Sciortino, S., Vaiana, G. S., Majer, P., Bookbinder, J., Golub, L., Harnden, F. R., \& Rosner, R. 1987, ApJ, 315, 687

Marcy, G. W., Butler, R. P., Vogt, S. S., Liu, M. C., Laughlin, G., Apps, K., Graham, J. R., Lloyd, J., Luhman, K. L., \& Jayawardhana, R. 2001, ApJ, 555, 418

McCarthy, C. \& Zuckerman, B. 2004, AJ, 127, 2871

McCaughrean, M. J., Close, L. M., Scholz, R.-D., Lenzen, R., Biller, B., Brandner, W., Hartung, M., \& Lodieu, N. 2004, A\&A, 413, 1029

Metchev, S. A. \& Hillenbrand, L. A. 2004, ApJ, 617, 1330

Mugrauer, M., Seifahrt, A., Neuhäuser, R., \& Mazeh, T. 2006, MNRAS, L101

Nakajima, T., Oppenheimer, B. R., Kulkarni, S. R., Golimowski, D. A., Matthews, K., \& Durrance, S. T. 1995, Nature, 378, 463

Oppenheimer, B. R., Golimowski, D. A., Kulkarni, S. R., Matthews, K., Nakajima, T., CreechEakman, M., \& Durrance, S. T. 2001, AJ, 121, 2189

Oppenheimer, B. R., Kulkarni, S. R., Matthews, K., \& Nakajima, T. 1995, Science, 270, 1478

Perryman, M. A. C. et al. 1997, A\&A, 323, L49

Preibisch, T. \& Feigelson, E. D. 2005, ApJS, 160, 390

Rayner, J. T., Toomey, D. W., Onaka, P. M., Denault, A. J., Stahlberger, W. E., Watanabe, D. Y., \& Wang, S. 1998, in Proc. SPIE: Infrared Astronomical Instrumentation, ed. A. M. Fowler, Vol. $3354,468-479$

Rebolo, R., Zapatero Osorio, M. R., Madruga, S., Bejar, V. J. S., Arribas, S., \& Licandro, J. 1998, Science, 282, 1309

Rocha-Pinto, H. J., Castilho, B. V., \& Maciel, W. J. 2002, A\&A, 384, 912

Rocha-Pinto, H. J. \& Maciel, W. J. 1996, MNRAS, 279, 447

Roche, P. F. et al. 2003, in Instrument Design and Performance for Optical/Infrared Ground-based Telescopes. Proceedings of the SPIE, ed. M. Iye \& A. F. M. Moorwood, Vol. 4841, 901-912

Santos, N. C., Israelian, G., \& Mayor, M. 2004, A\&A, 415, 1153

Santos, N. C., Israelian, G., Mayor, M., Bento, J. P., Almeida, P. C., Sousa, S. G., \& Ecuvillon, A. 2005, A\&A, 437, 1127

Saumon, D., Marley, M. S., Cushing, M. C., Leggett, S. K., Roellig, T. L., Lodders, K., \& Freedman, R. S. 2006, ApJ, 647, 552 
Schmitt, J. H. M. M. \& Liefke, C. 2004, A\&A, 417, 651

Simons, D. A. \& Tokunaga, A. 2002, PASP, 114, 169

Skumanich, A. 1972, ApJ, 171, 565

Soderblom, D. R., Duncan, D. K., \& Johnson, D. R. H. 1991, ApJ, 375, 722

Stern, R. A., Schmitt, J. H. M. M., \& Kahabka, P. T. 1995, ApJ, 448, 683

Strassmeier, K., Washuettl, A., Granzer, T., Scheck, M., \& Weber, M. 2000, A\&AS, 142, 275

Takeda, G., Ford, E. B., Sills, A., Rasio, F. A., Fischer, D. A., \& Valenti, J. A. 2006, ApJS, in press (astro-ph/0607235)

Tokunaga, A. T., Simons, D. A., \& Vacca, W. D. 2002, PASP, 114, 180

Udry, S., Mayor, M., Naef, D., Pepe, F., Queloz, D., Santos, N. C., \& Burnet, M. 2002, A\&A, 390, 267

Vacca, W. D., Cushing, M. C., \& Rayner, J. T. 2003, PASP, 115, 389

Valenti, J. A. \& Fischer, D. A. 2005, ApJS, 159, 141

Vrba, F. J. et al. 2004, AJ, 127, 2948

Walter, F. M. \& Berry, D. C. 1991, in The Sun in Time, ed. C. P. Sonett, M. S. Giampapa, \& M. S. Matthews (Tucson: University of Arizona Press), 653

Wilson, J. C., Kirkpatrick, J. D., Gizis, J. E., Skrutskie, M. F., Monet, D. G., \& Houck, J. R. 2001, AJ, 122, 1989

Wright, G. S., Mountain, C. M., Bridger, A., Daly, P. N., Griffin, J. L., \& Ramsay Howat, S. K. 1993, in Proc. SPIE Vol. 1946, p. 547-557, Infrared Detectors and Instrumentation, ed. A. M. Fowler, 547-557

Wright, J. T., Marcy, G. W., Butler, R. P., \& Vogt, S. S. 2004, ApJS, 152, 261 


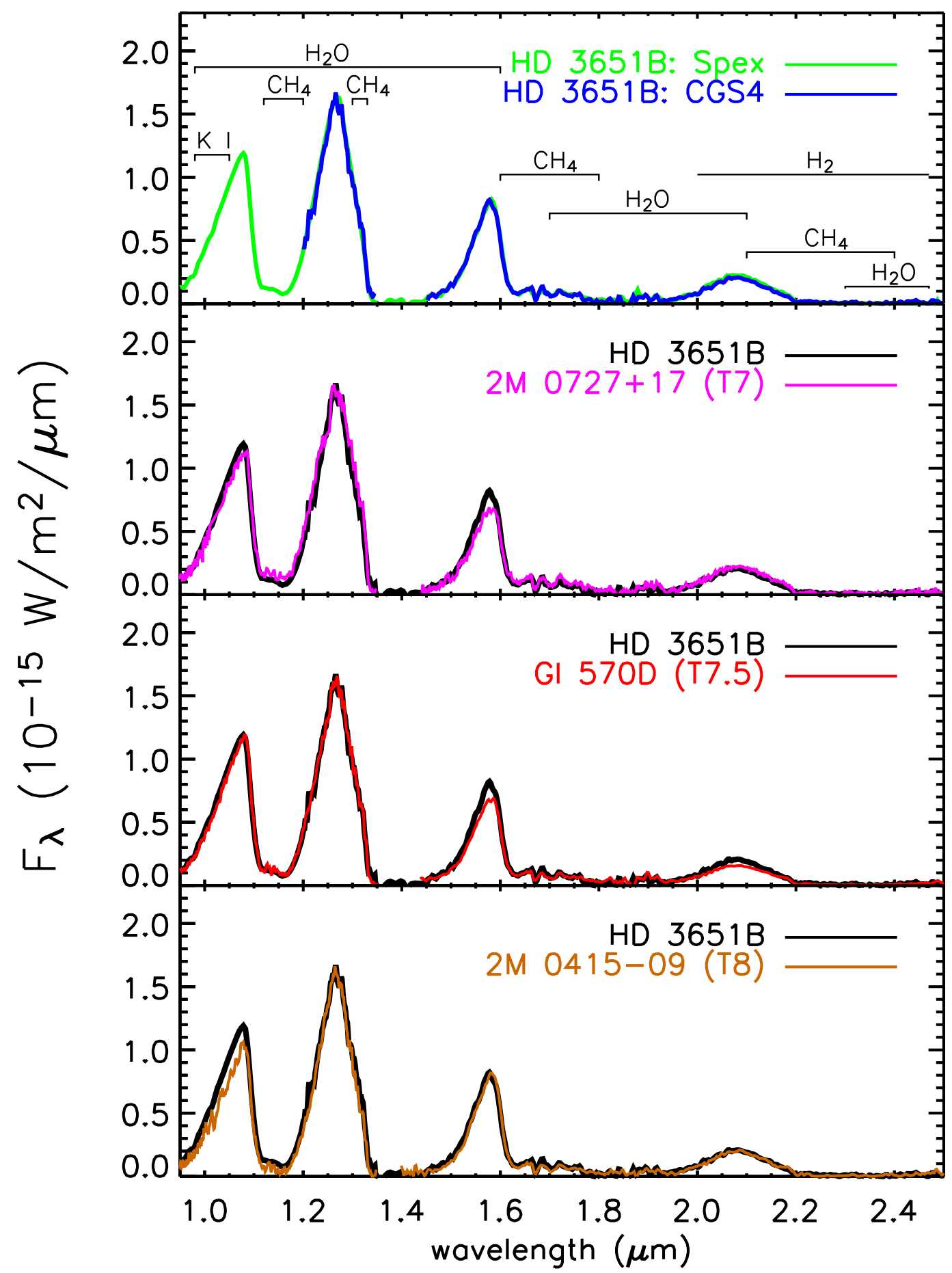

Fig. 1.- Top panel: Our near-IR spectra of HD 3651B obtained with UKIRT/CGS4 and IRTF/Spex. Other panels: Same spectra of HD 3651B plotted as a thick black line, with mostly the UKIRT/CGS4 data plotted and the IRTF/Spex data filling in the $<1.2 \mu \mathrm{m}$ and $1.35-$ $1.45 \mu \mathrm{m}$ regions. Spectra of other late-T dwarfs are plotted as colored lines. Their near-IR spectra $(>1 \mu \mathrm{m})$ were also obtained with CGS4, with the same intrumental setup and spectral resolution Geballe et al. 2001, 2002; Knapp et al. 2004), and the $<1 \mu \mathrm{m}$ spectra come from Burgasser et al. (2003). The spectra have been normalized by their peak flux. 


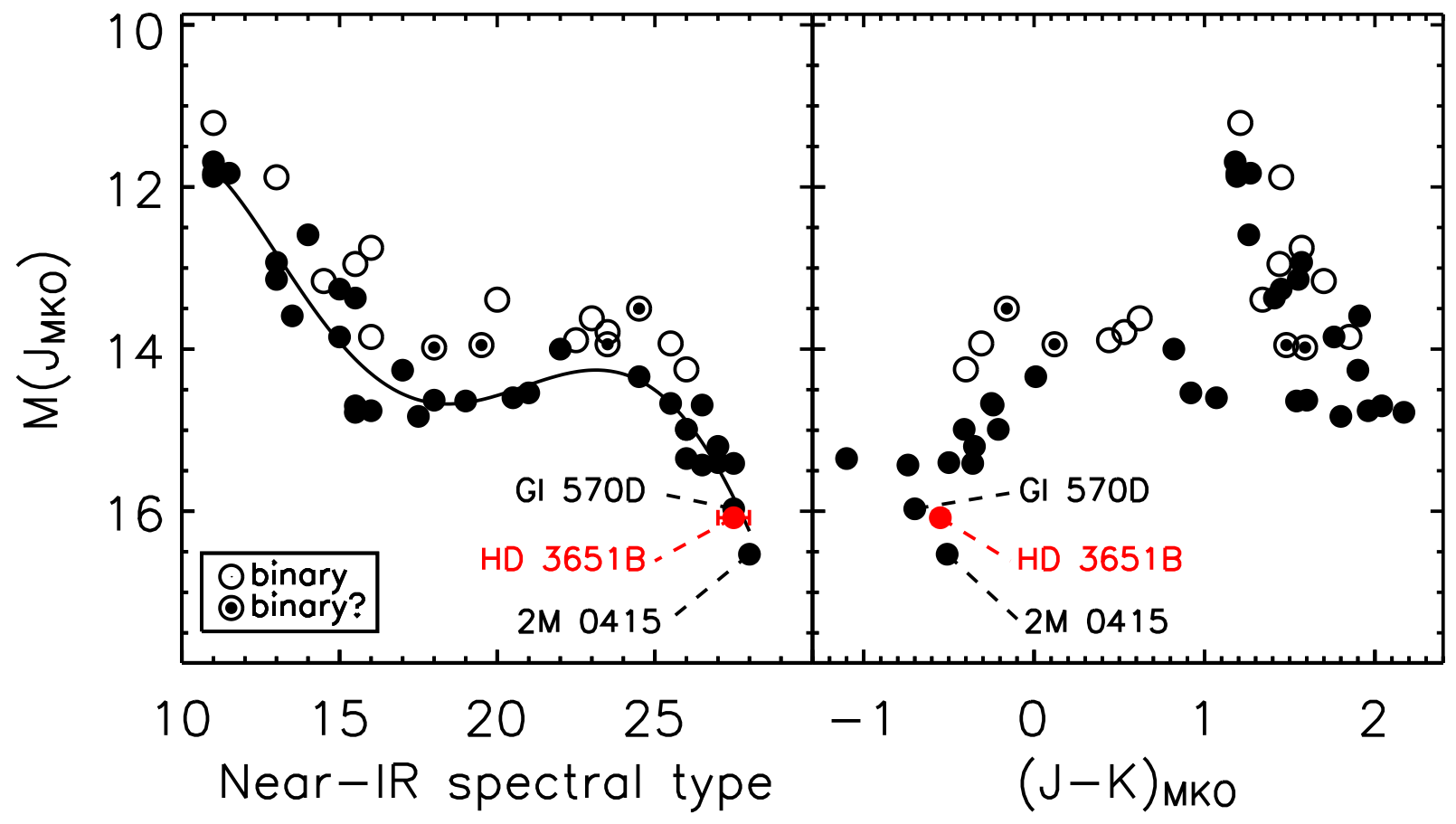

Fig. 2.- Near-IR photometry of HD 3651B compared to other L and T dwarfs with known distances, largely from Dahn et al. (2002) and Vrba et al. (2004) as tabulated by Knapp et al. (2004). Known binaries are plotted as open circles, and possible binaries as encircled dots. See Liu et al. (2006) for additional references and discussion of possible binaries. The photometric measurements use the MKO system, and their errors are comparable to or smaller than the symbol size. Left: $J$-band absolute magnitude as a function of near-IR spectral type and $J-K$ color. Spectral types are based on the Geballe et al. (2002) scheme for the L dwarfs and the Burgasser et al. (2006b) scheme for the T dwarfs, with L0 being 10, L1 begin 11, T0 being 20, etc. on the X-axis. The solid line shows a 5th-order polynomial fit, excluding known and possible binaries and HD 3651B. The T7.5 dwarf Gl 570D and the T8 dwarf 2MASS 0415-0935 are labeled. Right: $J$-band absolute magnitude versus $J-K$ color. 


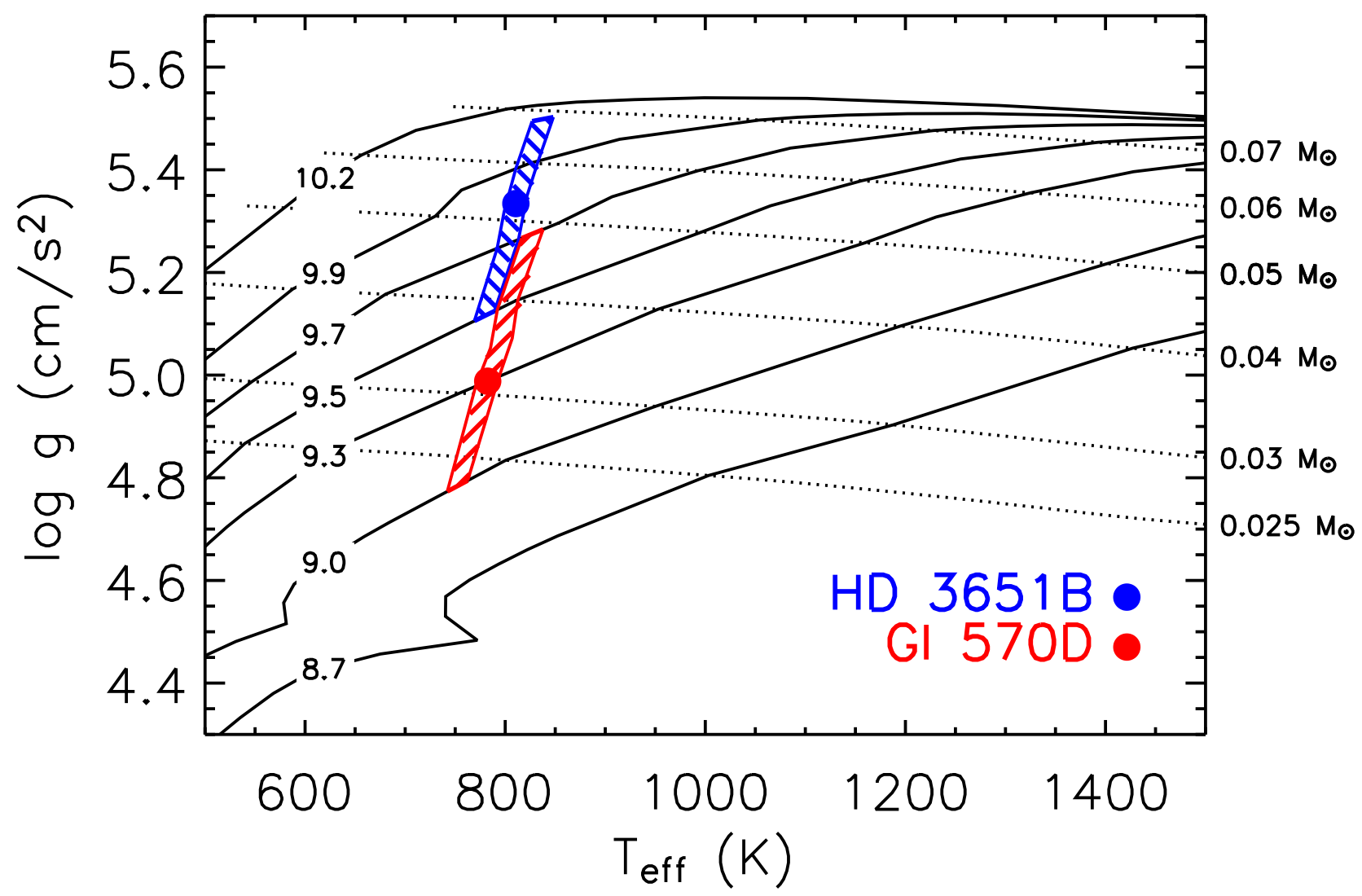

Fig. 3.- Inferred $T_{\text {eff }}$ and $\log (g)$ for HD $3651 \mathrm{~B}$ and Gl $570 \mathrm{D}$ based on sol,ar-metallicity evolutionary models by Burrows et al. (1997) and observational constraints summarized in Table 1. Solid lines are isochrones from 0.5 to 16 Gyr, labeled by the logarithm of their age. Dotted lines represent iso-mass models, labeled on the right side of the plot in units of $M_{\odot}$. The solid colored dots indicate the model-derived $T_{\text {eff }}$ and $\log (g)$ for HD $3651 \mathrm{~B}$ and Gl $570 \mathrm{D}$, given the computed $L_{\mathrm{bol}}$ 's and estimated ages of the objects. The $T_{\text {eff }}$ of the two objects are very similar, with HD 3651B having $\mathrm{a} \approx 0.3$ dex higher surface gravity. The colored hatched regions indicate the observational uncertainties. Note that the apparent overlap of the uncertainties in determining $\log (g)$ is somewhat misleading, since each colored region represents the uncertainty in the absolute ages; the relative ages of the primary stars is better constrained, with HD $3651 \mathrm{~B}$ being $\approx 3 \times$ older than Gl 570D $(\S 4.1)$. 


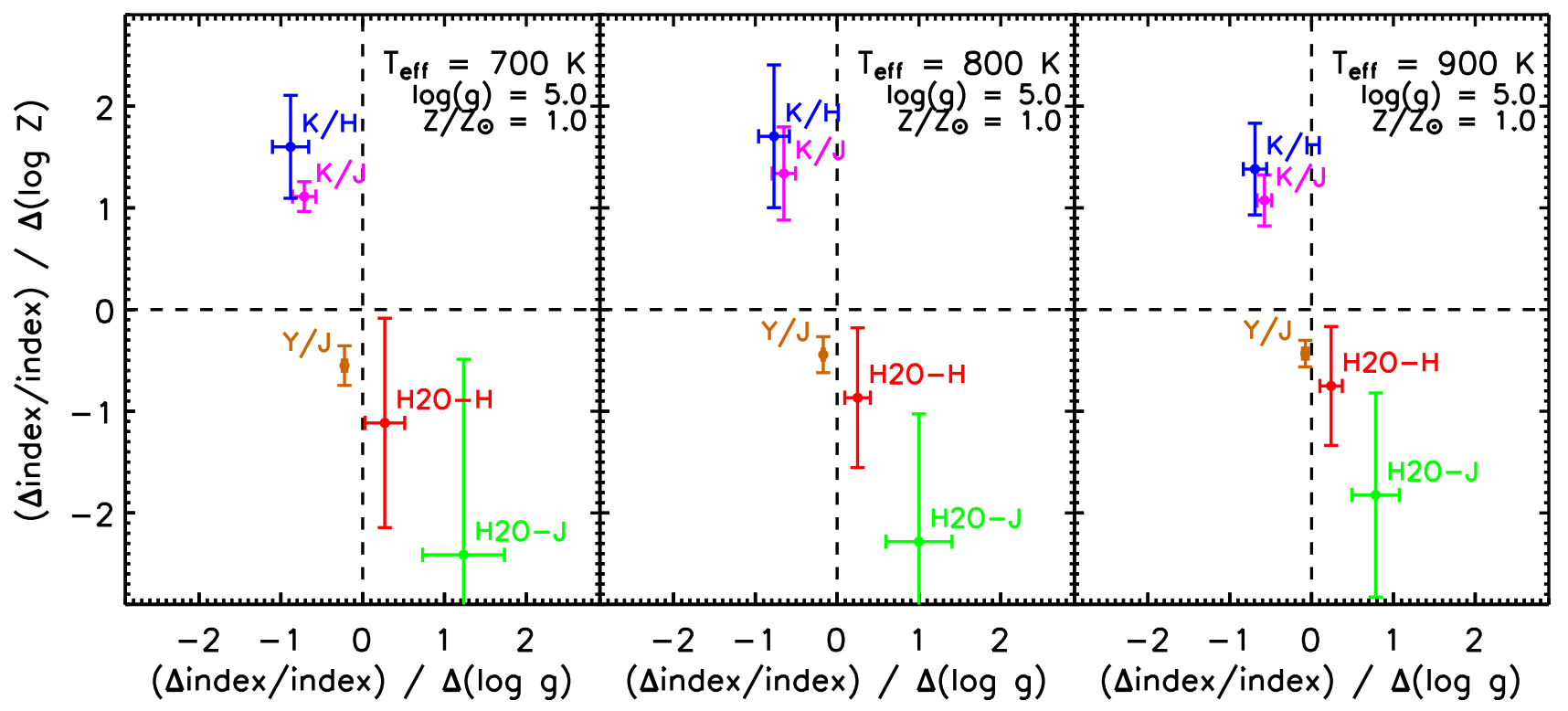

Fig. 4.- Quantitative representation of the metallicity and surface gravity sensitivity of the Burgasser et al. (2006a) spectral indices, based on condensate-free atmosphere models by Burrows et al. (2006). The models have been smoothed to a spectral resolution of $100 \AA$, appropriate for IRTF/Spex data (though the results are essentially the same for $10 \times$ higher spectral resolution). For each spectral index, the $x$-axis value gives its fractional change as $\log (g)$ is changed and the $y$-axis as $Z$ is changed, about a nominal reference value of $\log (g)=5.0$ and $Z / Z_{\odot}=1.0$. A purely metallicity-sensitive index would have a large ordinate value but an abscissa of zero (i.e., it would lie on the vertical line), while a purely gravity-sensitive index would lie on the horizontal line. The error bars show the range in the sensitivity of the indices as $\log (g)$ changes over the intervals $[4.5,5.0]$ and $[5.0,5.5]$ and as $Z / Z_{\odot}$ changes over the intervals $[0.3,1.0]$ and $[1.0,3.0]$. This plot indicates that the $K / H$ and $K / J$ indices are very sensitive to both changes in metallicity and surface gravity at fixed $T_{\text {eff }}$. 
Table 1. Properties of the Late-T Dwarf Companions HD 3651B and Gl 570D

\begin{tabular}{|c|c|c|}
\hline Property & HD 3651B & Gl 570D \\
\hline Spectral type & $\mathrm{T} 7.5 \pm 0.5$ & $\mathrm{~T} 7.5 \pm 0.5^{\mathrm{a}}$ \\
\hline Primary star spectral type & $\mathrm{K}_{0} \mathrm{~V}^{\mathrm{b}}$ & $\mathrm{K} 4 \mathrm{~V}^{\mathrm{b}}$ \\
\hline Distance (pc) & $11.11 \pm 0.09^{c}$ & $5.90 \pm 0.06^{\mathrm{c}}$ \\
\hline Estimated age (Gyr) & $6_{-3}^{+6 \mathrm{~d}}$ & $2_{-1}^{+3 \mathrm{~d}}$ \\
\hline$[\mathrm{Fe} / \mathrm{H}]$ & $0.09-0.16^{\mathrm{e}}$ & $0.01-0.10^{\mathrm{e}}$ \\
\hline$J$ (mags) & $16.31 \pm 0.03$ & $14.82 \pm 0.05^{\mathrm{f}}$ \\
\hline$H$ (mags) & $16.72 \pm 0.03$ & $15.28 \pm 0.05^{\mathrm{f}}$ \\
\hline$K$ (mags) & $16.86 \pm 0.03$ & $15.52 \pm 0.05^{\mathrm{f}}$ \\
\hline$J-H$ & $-0.41 \pm 0.04$ & $-0.46 \pm 0.07^{\mathrm{f}}$ \\
\hline$H-K$ & $-0.14 \pm 0.04$ & $-0.24 \pm 0.07^{\mathrm{f}}$ \\
\hline$J-K$ & $-0.55 \pm 0.04$ & $-0.70 \pm 0.07^{\mathrm{f}}$ \\
\hline$M_{J}(\mathrm{mags})$ & $16.08 \pm 0.03$ & $15.97 \pm 0.05^{\mathrm{f}}$ \\
\hline$M_{H}(\mathrm{mags})$ & $16.49 \pm 0.03$ & $16.43 \pm 0.05^{\mathrm{f}}$ \\
\hline$M_{K}$ (mags) & $16.63 \pm 0.03$ & $16.67 \pm 0.05^{\mathrm{f}}$ \\
\hline $\log \left(L_{\mathrm{bol}} / L_{\odot}\right)$ & $-5.58 \pm 0.05$ & $-5.53 \pm 0.05^{\mathrm{g}}$ \\
\hline Mass $\left(M_{\mathrm{Jup}}\right)$ & $56_{-16}^{+16}$ & $33_{-9}^{+18}$ \\
\hline$T_{\text {eff }}(\mathrm{K})$ & $810_{-30}^{+30}$ & $780_{-30}^{+45}$ \\
\hline $\log (g)(\operatorname{cgs})$ & $5.3_{-0.2}^{+0.2}$ & $5.0_{-0.2}^{+0.3}$ \\
\hline
\end{tabular}

Note. - The tabulated results are from this paper, unless otherwise noted. All photometry is on the MKO system.

Burgasser et al. (2006b)

${ }^{\mathrm{b}}$ From SIMBAD.

${ }^{c}$ Parallax for the primary star from Perryman et al. (1997).

${ }^{\mathrm{d}}$ See $\S 3.2, \S 4.1$, and Table 3 .

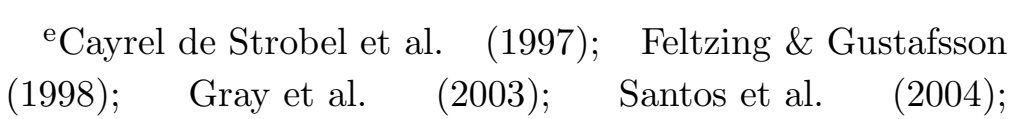

Valenti \& Fischer (2005); Santos et al. (2005)

fGeballe et al. (2001) 
golimowski et al. (2004) 
Table 2. Spectral Typing of HD 3651B

\begin{tabular}{cccccc}
\hline \hline Dataset & $\mathrm{H}_{2} \mathrm{O}-J$ & $\mathrm{CH}_{4}-J$ & $\mathrm{H}_{2} \mathrm{O}-H$ & $\mathrm{CH}_{4}-H$ & $\mathrm{CH}_{4}-K$ \\
\hline UKIRT/CGS4 & $\ldots$ & $0.214(\mathrm{~T} 7.5)$ & $0.212(\mathrm{~T} 7.5)$ & $0.128(\mathrm{~T} 7.5)$ & $0.082(\geq \mathrm{T} 7)$ \\
IRTF/Spex & $0.058(\mathrm{~T} 8)$ & $0.192(\mathrm{~T} 8)$ & $0.197(\mathrm{~T} 7.5)$ & $0.121(\mathrm{~T} 8)$ & $0.059(\geq \mathrm{T} 7)$ \\
\hline
\end{tabular}

Note. - Measurements of spectral indices along with the corresponding spectral type in parentheses based on the Burgasser et al. (2006b) classification scheme.

Table 3. Age Estimates for HD 3651A and Gl 570A

\begin{tabular}{lcccc}
\hline \hline \multicolumn{1}{c}{ Method } & Ref & $\begin{array}{c}\text { HD 3651A } \\
(\mathrm{Gyr})\end{array}$ & $\begin{array}{c}\text { Gl 570A } \\
(\mathrm{Gyr})\end{array}$ & $\begin{array}{c}\text { Relative age } \\
\text { HD 3651A / Gl 570A }\end{array}$ \\
\hline CaII HK emission & 1,2 & $5-9$ & $0.8-2.2$ & $\approx 5$ \\
X-ray emission & 3 & $0.9-2.2$ & $0.4-0.8$ & $\approx 2.5$ \\
Rotation period & 4,5 & 15 & 6 & 2.5 \\
Isochrones & 6 & $8_{-5}^{+4}$ & $3.3_{-3.1}^{+8.3}$ & $\approx 2.5$ \\
Isochrones & 7 & $>11.8$ & $<0.6$ & $>20$ \\
\hline
\end{tabular}

Note. - Age estimates for each star are discussed in $\S 3.2$ and $\S 4.1$. The relationship used to convert the observed quantity (e.g., X-ray emission) to the stellar age is given in the cited reference. In computing the relative ages in the last column, the geometric mean is used for methods that produce an age range.

References. - (1) Donahue (1993); (2) Donahue (1998); (3) Gaidos (1998); (4) Skumanich (1972); (5) Lachaume et al. (1999); (6) Valenti \& Fischer (2005); (7) Takeda et al. (2006) 
Table 4. Spectral Index Measurements for Gl 570D and HD 3651B

\begin{tabular}{lccc}
\hline \hline \multirow{2}{*}{ Index } & \multirow{2}{*}{$\begin{array}{c}\text { Gl 570D } \\
\text { Observed }\end{array}$} & \multicolumn{2}{c}{ HD 3651B } \\
& Observed & Predicted \\
\hline $\mathrm{H}_{2} \mathrm{O}-J$ & $0.066 \pm 0.005$ & $0.058( \pm 0.003)$ & $0.087_{-0.006}^{+0.006}$ \\
$\mathrm{H}_{2} \mathrm{O}-H$ & $0.204 \pm 0.005$ & $0.205 \pm 0.011$ & $0.237_{-0.010}^{+0.010}$ \\
$Y / J$ & $0.412 \pm 0.025$ & $0.449( \pm 0.027)$ & $0.387_{-0.007}^{+0.007}$ \\
$K / J$ & $0.110 \pm 0.008$ & $0.142 \pm 0.008$ & $0.116_{-0.013}^{+0.014}$ \\
$K / H$ & $0.250 \pm 0.005$ & $0.292 \pm 0.022$ & $0.276_{-0.040}^{+0.043}$ \\
\hline
\end{tabular}

Note. - Measurements of Burgasser et al. (2006a) spectral indices using our spectra for HD 3651B and spectra from Geballe et al. (2001) and Burgasser et al. (2006a) for Gl 570D. For the second and third columns, the error bars are computed from the standard deviation of the UKIRT/CGS4 and IRTF/Spex measurements. Where only Spex data are available, we assume an error of $6 \%$, based on the median errors of the other indices, and place it in parentheses. The last column gives the modelpredicted values for HD 3651B, assuming a $0.10 \pm 0.05$ dex higher metallicity, a $0.25 \pm 0.05$ dex higher surface gravity, and $20 \pm 10 \mathrm{~K}$ hotter temperature than Gl 570D. See $\S 4.2$ for a full explanation. 
Table 5. Sensitivity of Spectral Indices to Surface Gravity, Metallicity, and Effective Temperature for $T_{\text {eff }}=800 \mathrm{~K}, \log (g)=5.0(\mathrm{cgs})$, and $Z / Z_{\odot}=1.0$

\begin{tabular}{lcccccc}
\hline \hline \multirow{2}{*}{ Spectral Index } & \multicolumn{2}{c}{$(\Delta$ (index $) /$ index $) / \Delta(\log g)$} & \multicolumn{2}{c}{$(\Delta$ (index $) /$ index $) / \Delta(\log Z)$} & \multicolumn{2}{c}{$(\Delta($ index $) /$ index $) / \Delta\left(T_{\text {eff }} / 100 \mathrm{~K}\right)$} \\
& {$[4.5,5.0]$} & {$[5.0,5.5]$} & {$[-0.5,0.0]$} & {$[0.0,+0.5]$} & {$[700 \mathrm{~K}, 800 \mathrm{~K}]$} & {$[800 \mathrm{~K}, 900 \mathrm{~K}]$} \\
\hline $\mathrm{H}_{2} \mathrm{O}-J$ & 0.60 & 1.40 & -3.54 & -1.03 & 0.36 & 0.47 \\
$\mathrm{H}_{2} \mathrm{O}-H$ & 0.09 & 0.41 & -1.55 & -0.18 & 0.42 & 0.37 \\
$Y / J$ & -0.16 & -0.18 & -0.62 & -0.27 & 0.10 & 0.06 \\
$K / J$ & -0.79 & -0.51 & 0.88 & 1.80 & 0.17 & 0.14 \\
$K / H$ & -0.96 & -0.58 & 1.00 & 2.41 & 0.22 & 0.21 \\
\hline
\end{tabular}

Note. - Dependence of Burgasser et al. (2006b) spectral indices as a function of surface gravity, metallicity, and effective temperature, computed from condensate-free models by Burrows et al. (2006) for objects around the reference values of $T_{\text {eff }}=800 \mathrm{~K}$ and $\log (g)=5.0$ and $Z / Z_{\odot}=1.0$. The models have been smoothed to a spectral resolution of $100 \AA$, appropriate for IRTF/Spex data, though the results are essentially the same for $10 \times$ higher spectral resolution $(\lesssim 0.01$ difference in values). Each column gives the fractional change in the spectral index as the parameters $\log (g), Z$, and $T_{\text {eff }}$ are changed about the reference values, with the change in the parameter listed in the second row of headings. For example, the first row of data shows that in the models the $\mathrm{H}_{2} \mathrm{O}-J$ index increases by $0.6 \times 0.5=30 \%$ as $\log (g)$ changes from 4.5 to 5.0 , decreases by $3.54 \times 0.5=180 \%$ as $\log (Z)$ changes from -0.5 to 0.0 , and increases by $0.36 \times 100 / 100=36 \%$ as $T_{\text {eff }}$ changes from $700 \mathrm{~K}$ to $800 \mathrm{~K}$. Spectral indices with larger positive (negative) values have stronger (anti-)correlations with $\log (g), Z$, or $T_{\text {eff }}$. 